"RASEEENDER Trascender, Contabilidad y Gestión Núm. 9 (septiembre - diciembre del 2018). ISSN: 2448-6388. Universidad de Sonora. Departamento de Contabilidad. Reserva de Derechos 04-2015-04172070800-203.

\title{
La Auditoría Financiera de Gabinete como instrumento de evaluación de la Cuenta Pública Municipal en Sonora
}

The Cabinet Financial Audit as an instrument for assessing the Municipal Public Account in Sonora

$$
\text { Luz Elena Valenzuela Valdez }{ }^{1}
$$

\section{Resumen}

A través de los diferentes tipos de Auditoría y Fiscalización, se previenen y combaten irregularidades en el uso de los recursos públicos establecidos en sus presupuestos ya sea de ingresos o de egresos, así como también la administración de la hacienda, el patrimonio y la deuda pública. Este informe está enfocado a describir el proceso de la Auditoría Financiera de Gabinete como instrumento de Evaluación de la Cuenta Pública Municipal 2017 de los Municipios del Estado de Sonora.

La Cuenta Pública Municipal es el informe que los entes públicos municipales a través de los ayuntamientos, que rinden a la legislatura local, sobre su gestión financiera a efecto de comprobar que la recaudación, administración, manejo y custodia, así como la aplicación de los recursos públicos durante el ejercicio fiscal comprendido del $1^{\circ}$ de enero al 31 de diciembre de 2017, se ejercieron en los términos de las disposiciones legales y administrativas aplicables. El contenido de dicho informe deberá presentarse en apego a lo establecido en la Ley de General de Contabilidad Gubernamental y demás normatividad vigente.
El Instituto Superior de Auditoría y Fiscalización se constituye como el organismo público autónomo, encargado de revisar y fiscalizar la cuenta pública municipal, en los términos establecidos en esta Constitución y demás legislaciones inherentes a la ejecución de la función de auditoría, además de implementar acciones de prevención en materia de corrupción.

Entonces podemos definir a la Auditoría Financiera de Gabinete, como la revisión de la Situación Económica, las Finanzas Públicas, la Deuda Pública, los Activos y el Patrimonio Municipal informados en la Cuenta Pública Municipal, además del cumplimiento legal de la presentación de la información contable, presupuestal, programática y demás anexos, con la finalidad de conocer la calidad de la información financiera y que su elaboración haya sido de conformidad con lo establecido en la Ley General de Contabilidad Gubernamental.

Palabras clave: auditoría, fiscalización, cuenta pública, municipios.

DOI: https://doi.org/10.36791/tcg.v0i9.40

Recibido: 15 de junio de 2018.

Aceptado: 12 de agosto de 2018.

\footnotetext{
${ }^{1}$ Luz Elena Valenzuela Valdez. Contador Público. Auditor supervisor del ISAF (Instituto Sonorense de Auditoria Fiscal). Correo: levv76@hotmail.com
} 


\section{Abstract}

Through the different types of Audit and Control, irregularities are prevented and combated in the use of public resources established in their budgets, whether of income or expenditure, as well as the administration of the treasury, the patrimony and the public debt. This report is focused on describing the process of the Cabinet Financial Audit as an instrument of Evaluation of the Municipal Public Account 2017 of the Municipalities of the State of Sonora.

The Municipal Public Account is the report that the municipal public entities through the city councils, that yield to the local legislature, on its financial management in order to verify that the collection, administration, management and custody, as well as the application of the resources During the fiscal year comprised from January 1 to December 31, 2017, they were exercised under the terms of the applicable legal and administrative provisions. The content of said report must be submitted in accordance with the provisions of the General Government Accounting Law and other current regulations.
The Higher Institute of Auditing and Auditing is constituted as the autonomous public body, in charge of reviewing and auditing the municipal public account, in the terms established in this Constitution and other legislation inherent to the execution of the audit function, in addition to implementing actions of prevention of corruption.

Then we can define the Cabinet Financial Audit, such as the review of the Economic Situation, Public Finances, Public Debt, Assets and Municipal Heritage informed in the Municipal Public Account, in addition to the legal compliance of the presentation of the accounting information , budgetary, programmatic and other annexes, in order to know the quality of financial information and that its preparation has been in accordance with the provisions of the General Law of Government Accounting.

Keywords: audit, public account, municipalities. 


\section{Introducción}

El tema de la auditoria y fiscalización gubernamental ha cobrado importancia en la última década, no sólo por considerarse un mecanismo de control en la administración pública sino también como parte fundamental de la rendición de cuentas, además de convertirse en una herramienta de legitimidad para los gobiernos, ya que es a través de ella como la Entidad Estatal de Fiscalización Superior evalúa la situación financiera que guarda la hacienda pública del gobierno en todos sus niveles; presenta los resultados obtenidos para que estos sean analizados, corregidos y de ser necesario aplicar las sanciones que dictaminen las autoridades correspondientes.

La auditoría y fiscalización gubernamental es uno de los instrumentos utilizados para evaluar la gestión pública, es decir que esta se realice con economía, eficiencia, eficacia y transparencia, de acuerdo a los lineamientos y disposiciones legales vigentes, de ahí la importancia de conocer la normatividad básica metodológica para llevar a cabo este proceso.

Este trabajo está enfocado a destacar la importancia de la Auditoría Financiera de Gabinete como instrumento de revisión, análisis y evaluación de la información que contiene la Cuenta Pública 2017, de los municipios del Estado de Sonora.

\section{Antecedentes}

Pineda Pablos, García Figueroa, \& Rodríguez Camou ( 2007:210) refieren que la revisión de la cuenta pública representa un papel central en el proceso de rendición de cuentas, y la calidad con la que se haga este proceso, denota en el avance de la rendición de cuentas en México, aun cuando existen otros mecanismos de evaluación y fiscalización; la cuenta pública es considerada como el proceso formal e institucional de informar el estado que guardan la hacienda pública del Gobierno en sus tres niveles.

Según Sour (2011:91) hoy México tiene en sus manos una oportunidad única para que el sistema de contabilidad gubernamental mejore la transparencia y la rendición de cuentas en México. Con la aprobación de la Ley General de Contabilidad Gubernamental (LGCG) en diciembre del 2008, cuyo objetivo es el de facilitar el registro, control y la fiscalización de los activos, pasivos, ingresos, deuda y gasto en los diferentes niveles de gobierno de México. La LGCG además busca desarrollar la unión entre el presupuesto y la contabilidad.

Para Sour (2017:421) el Consejo Nacional de Armonización Contable (CONAC) nace en conjunción con la LGCG como órgano encargado de la coordinación para la armonización de la contabilidad gubernamental. El CONAC agrupa a representantes de las entidades federativas, del gobierno federal y de los municipios con el propósito de señalar los elementos mínimos de los manuales de contabilidad, emitir los reclasificadores de los catálogos de cuentas, las normas contables para la emisión de la información financiera, generar los lineamientos para el establecimiento de un sistema de costos, establecer el marco metodológico para la integración entre los registros contables y el proceso presupuestario y definir las disposiciones para el registro contable del esquema de deuda pública.

Los datos contables permitirán evaluar los alcances obtenidos en relación a la eficiencia y eficacia que cada ente público observe en el desempeño de sus funciones y atribuciones que la ley les confiera. Por tanto la aplicación de la contabilidad gubernamental es el sendero a seguir para dar a conocer los resultados del ejercicio del gasto. De ahí que la cuenta pública permite a la Entidad Estatal de Fiscalización Superior revisar, analizar y evaluar su contenido y durante ese proceso de fiscalización determinar los hallazgos preliminares, e informarlos a los representantes de cada ente público.

Este trabajo se lleva a cabo mediante auditorias ya sea de gabinete o fiscalización de campo, en el ámbito legal, financiero, presupuestal, de desempeño, obra pública o forense. 


\section{Cuenta Pública Municipal}

Existen múltiples métodos y herramientas para evaluar la rendición de cuentas de la Administración Municipal, entre ellas destaca la cuenta pública que cada año se entrega a la legislatura correspondiente.

La cuenta pública es un informe anual previsto por las leyes mexicanas para que los gobiernos informen sobre el origen de sus ingresos, el destino de sus gastos y las razones de sus decisiones; así como para que el poder legislativo realice su función de fiscalización y vigilancia de los fondos públicos. (Pineda Pablos et al. 2007:210).

March y Olsen $(1995,150)$ refieren que la finalidad de las cuentas públicas en las democracias modernas es presentar "una historia de la intención humana y agencia que envuelven a los funcionarios públicos y a los políticos, y que conducen a que estos individuos sean responsables". (Lagunes López \& Pineda Pablos, 2013: 236).

La Ley de Fiscalización Superior para el Estado de Sonora, establece que la Cuenta Pública es el informe que los poderes del Estado y los entes públicos estatales presentarán de manera individual para ser consolidada a través del Ejecutivo estatal; los municipios de la Entidad y los entes públicos municipales a través de los ayuntamientos, que rinden a la legislatura local, sobre su gestión financiera a efecto de comprobar que la recaudación, administración, manejo y custodia, así como la aplicación de los recursos públicos durante el ejercicio fiscal comprendido del $1^{\circ}$ de enero al 31 de diciembre de cada año, se ejercieron en los términos de las disposiciones legales y administrativas aplicables. (Art. 22)

Tomando como referencia la normatividad vigente del Consejo de Armonización Contable (CONAC) en el Acuerdo por el que se armoniza la estructura de las cuentas públicas, cuya última reforma fue publicada en el DOF 29-02-2016, la Cuenta Pública del Ayuntamiento del Municipio será formulada e integrada por la Tesorería Municipal, y deberán presentarlas al Poder Legislativo de la Entidad Federativa que corresponda, dentro del plazo establecido en la normatividad local vigente, así como publicarla en la página de Internet del Ayuntamiento del Municipio. Una vez que se entrega al Congreso del Estado, este la turnara de forma inmediata a la Entidad Estatal de Fiscalización Superior para la revisión correspondiente a su competencia.

La Ley General de Contabilidad Gubernamental en su Artículo 48, establece los requisitos mínimos de información contable y presupuestaria que deben elaborar los Ayuntamientos, en la misma temática a nivel estatal la Ley de Fiscalización para el Estado de Sonora (LFES) detalla estos requisitos en el Artículo 22.

El Instituto Superior de Auditoria y Fiscalización (ISAF) emite los lineamientos que establecen el contenido y formularios para la elaboración de la Cuenta Pública Municipal 2017, los cuales están basados en la LGCG, LFES, Ley de Disciplina Financiera de las Entidades Federativas y los Municipios y los lineamientos emitidos por el Consejo Nacional de Armonización Contable (CONAC). Cuyo contenido es el siguiente:

Oficio de Remisión

Anexo C.P.1: Balanza de Comprobación

Anexo C.P.2: Estado de Situación Financiera

Relaciones Analíticas

Conciliaciones Bancarias

Anexo C.P.3: Estado de Variación en la Hacienda Pública

Anexo C.P.4: Estado de Cambios en la Situación Financiera

Anexo C.P.5: Estado Analítico del Activo 
Anexo C.P.6: Estado de Actividades

Notas a los estados financieros

Justificación Global al Ahorro o Desahorro obtenido en el Ejercicio

Anexo C.P.7: Estado de Flujos de Efectivo

Anexo C.P.8: Estado Analítico de Ingresos

Anexo C.P.8A: Estado Analítico de Ingresos de Ingresos por Fuente de Financiamiento

Anexo C.P.9: Estado Analítico del Ejercicio del Presupuesto de Egresos por Objeto del Gasto (Capítulo y Concepto)

Anexo C.P.9A: Estado Analítico del Ejercicio del Presupuesto de Egresos Clasificación Económica (Por Tipo de Gasto)

Anexo C.P.10: Estado Analítico del Ejercicio del Presupuesto de Egresos Clasificación Administrativa

Anexo C.P.11: Desglose del Capítulo 40000 Transferencias, Asignaciones, Subsidios y Otras Ayudas

Anexo C.P.12: Avance Físico Financiero de los Programas de Inversión

Anexo C.P.13: Desglose de Gastos Efectuados con Recursos del Fondo de Aportaciones para la Infraestructura Social Municipal y de las Demarcaciones Territoriales del Distrito Federal con justificaciones

Anexo C.P.14: Desglose de Gastos Efectuados con Recursos del Fondo de Aportaciones para el Fortalecimiento Municipal y de las Demarcaciones Territoriales del Distrito Federal con justificaciones
Anexo C.P.15: Informe sobre la Situación de la Deuda Pública Municipal

Anexo C.P.16: Información Programática Presupuestal

Anexo C.P.16A: Estado Analítico del Ejercicio del Presupuesto de Egresos Clasificación Funcional (Finalidad y Función)

Anexo C.P. 17: Informe de Aplicación de Transferencias y Aportaciones Recibidas del Estado o Cualquier Otra Entidad Ajena al Ayuntamiento o de la Comunidad

Anexo C.P.18: Informe de Efectos Económicos y Sociales

Formatos de Ley de Disciplina Financiera:

Formato 2: Informe Analítico de la Deuda Pública y Otros Pasivos

Formato 3: Informe Analítico de Obligaciones Diferentes de Financiamiento

Formato 4: Balance Presupuestario

Formato 5: Estado Analítico de Ingresos Detallado

Formato 6a): Estado Analítico del Ejercicio del Presupuesto de Egresos Detallado Clasificación por Objeto del Gasto (Capítulo y Concepto)

Formato 6b): Estado Analítico del Ejercicio del Presupuesto de Egresos Detallado Clasificación Administrativa

Formato 6c): Estado Analítico del Ejercicio del Presupuesto de Egresos Detallado Clasificación Funcional (Finalidad y Función) 
Formato 6d): Estado Analítico del Ejercicio del Presupuesto de Egresos Detallado Clasificación de Servicios Personales por Categoría

La Constitución Política del Estado de Sonora establece que los poderes del Estado, los ayuntamientos y demás entidades públicas, independientemente de su naturaleza, son sujetos de fiscalización y estarán obligados, en los términos de esta Constitución y la ley, a rendir cuentas del ejercicio presupuestal que les corresponda.

\section{Auditoria Y Fiscalización De La Cuenta Pública}

En México, el tema de la fiscalización ha cobrado relevancia a partir del siglo XXI, y no sólo por su importancia como mecanismo de control en la administración pública federal y medio para el fortalecimiento de la rendición de cuentas, sino también como una herramienta en nuestra Moderna democracia, elemento coadyuvante de la legitimidad de los gobiernos, ya que es a través de ella y sus resultados como la ciudadanía está empezando a exigir gobiernos eficientes y transparentes. (Acosta Villada, 2013)

El Instituto Superior de Auditoría y Fiscalización se constituye como un organismo público autónomo, encargado de la revisión y fiscalización de los estados financieros y cuentas públicas estatal y municipal, en los términos establecidos en la Constitución local y la ley de la materia, también implementará acciones de prevención en materia de corrupción. La función de fiscalización será ejercida conforme a los principios de legalidad, definitividad, imparcialidad y confiabilidad. (Art. 67).

Las Normas Profesionales de Auditoría del Sistema Nacional de Fiscalización (NPASNF) constituyen un marco de referencia para la actuación de aquellos entes encargados de revisar la gestión de los recursos públicos, y señalan líneas y directrices que constituyen las mejores prácticas en la materia. (ASF, 2014)

Las NPASNF están basadas en las Normas Internacionales de las Entidades Fiscalizadoras Superiores (ISSAIs, por sus siglas en inglés), por ser éstas últimas un cuerpo sistematizado de preceptos resultado de la participación de especialistas de todos los países del mundo, pertenecientes no sólo a las Entidades Fiscalizadoras Superiores, sino también a Auditores Externos y Contralores Internos, así como las asociaciones profesionales que los representan Instituto de Auditores Internos (IIA), Federación Internacional de Contadores Públicos (IFAC)-, a través de un proceso ordenado y transparente para su creación, corrección y ampliación.

Estas características permiten que dichas Normas gocen de un consenso universal entre la comunidad auditora, por lo que constituyen una referencia necesaria para esta labor.

La Ley de Fiscalización Superior para el Estado de Sonora, establece que la revisión, auditoría y fiscalización de las cuentas públicas tiene por objeto:

I.- Evaluar los resultados de la gestión financiera:

La ejecución de la Ley de Ingresos y el ejercicio del Presupuesto de Egresos para verificar la forma y términos en que los ingresos fueron recaudados, obtenidos, captados y administrados; constatar que los recursos provenientes de financiamientos y otras obligaciones y empréstitos se contrataron, recibieron y aplicaron de conformidad con lo aprobado; y revisar que los egresos se ejercieron en los conceptos y partidas autorizados, incluidos, entre otros aspectos, la contratación de servicios y obra pública, las adquisiciones, arrendamientos, subsidios, aportaciones, donativos, transferencias, aportaciones a fondos, fideicomisos y demás instrumentos 
financieros, así como cualquier esquema o instrumento de pago a largo plazo; Para el caso de los empréstitos, que estos se ejerzan de conformidad con lo dispuesto por el artículo 117 de la Constitución Política de los Estados Unidos Mexicanos.

Si se cumplió con las disposiciones jurídicas aplicables en materia de sistemas de registro $\mathrm{y}$ contabilidad gubernamental; contratación de servicios, obra pública, adquisiciones, arrendamientos, conservación, uso, destino, afectación, enajenación y baja de bienes muebles e inmuebles; almacenes y demás activos; recursos materiales, y demás normatividad aplicable al ejercicio del gasto público;

Si la captación, recaudación, administración, custodia, manejo, ejercicio y aplicación de recursos, incluyendo subsidios, transferencias y donativos, y si los actos, contratos, convenios, mandatos, fondos, fideicomisos, prestación de servicios públicos, operaciones o cualquier acto que las entidades fiscalizadas, celebren o realicen, relacionados con el ejercicio del gasto público estatal, se ajustaron a la legalidad, y si no han causado daños o perjuicios, o ambos, en contra de la Hacienda Pública o, en su caso, del patrimonio de los entes públicos de los sujetos fiscalizados;

Comprobar si el ejercicio de la Ley de Ingresos y el Presupuesto de Egresos se ha ajustado a los criterios señalados en los mismos:

1. Si las cantidades correspondientes a los ingresos y a los egresos, se ajustaron o corresponden a los conceptos y a las partidas respectivas;

2. Si los programas y su ejecución se ajustaron a los términos y montos aprobados en el Presupuesto de Egresos; y

3. Si los recursos provenientes de financiamientos y otras obligaciones se obtuvieron en los términos autorizados y se aplicaron con la periodicidad y forma establecidas por las leyes y demás disposiciones aplicables, y si se cumplieron los compromisos adquiridos en los actos respectivos;

II.- Verificar el cumplimiento de los objetivos y metas contenidos en los programas:

Realizar auditorías del desempeño de los programas, verificando la eficiencia, la eficacia y la economía en el cumplimiento de los objetivos de los mismos;

Si se cumplieron las metas de los indicadores aprobados en el Presupuesto de Egresos y si dicho cumplimiento tiene relación con el Plan Estatal o municipales de Desarrollo, según corresponda y los programas respectivos, $\mathrm{y}$

Si se cumplieron los objetivos de los programas y las metas de gasto que promuevan la igualdad entre mujeres y hombres;

III.- Promover las acciones o denuncias correspondientes para la imposición de las sanciones administrativas y penales por las faltas graves que se adviertan derivado de sus auditorías e investigaciones, así como dar vista a las autoridades competentes cuando detecte la comisión de faltas administrativas no graves para que continúen la investigación respectiva y promuevan la imposición de las sanciones que procedan; $y$

IV.- Las demás que formen parte de la fiscalización de la Cuenta Pública o de la revisión del cumplimiento de los objetivos de los programas. (Art. 25)

Sobre la información financiera gubernamental y de la cuenta pública, la Ley General de Contabilidad Gubernamental subraya cuáles deben ser los criterios y los atributos que se asocian a ellos que deben tener los documentos de este carácter. Los criterios son cinco y los atributos relacionados son siete: Los estados financieros y la información emanada de la 
contabilidad deberán sujetarse a criterios de utilidad, confiabilidad, relevancia, comprensibilidad y de comparación, así como a otros atributos asociados a cada uno de ellos, como oportunidad, veracidad, representatividad, objetividad, suficiencia, posibilidad de predicción e importancia relativa, con el fin de alcanzar la modernización y armonización que la Ley determina (Art. 44)

Las exigencias de regularidad en el sector público rara vez tienen menos importancia para la rendición de cuentas que la expresión de una opinión sobre los estados financieros con amplias concesiones respecto de la materialidad. El objetivo de las NIA es permitir al auditor expresar una opinión sobre si los estados financieros han sido elaborados, en todos sus aspectos significativos, de acuerdo con un marco de información financiera determinado. El objetivo de fiscalización de una EFS es, por tanto, más amplio que los objetivos de una auditoría realizada con arreglo a las NIA. Además, el usuario de un informe de auditoría sobre estados financieros en el sector público busca un mayor grado de rendición de cuentas que el implicado por las NIA. (López Hernández A. M., 2013:17)

Para la ejecución de la auditoria es necesario realizar un plan global de auditoría que incluya conocer a la entidad que se va a auditar, establecer un objetivo de auditoría, determinar el alcance de auditoría, comprender el sistema de control interno, diseñar procedimientos adecuados que finalmente se condensará todo en el programa de auditoría. En la ejecución, el auditor debe de obtener toda la evidencia necesaria que fundamente los resultados y apoyen a la opinión. El juicio del auditor respecto de qué es evidencia suficiente apropiada de auditoría es influenciado por factores como: la evaluación del auditor de la naturaleza y nivel del riesgo inherente tanto a nivel de los estados financieros como a nivel del saldo de la cuenta o clase de transacciones, naturaleza de los sistemas de contabilidad y de control interno y la evaluación del riesgo de control, importancia relativa de la partida que se examina, experiencia ganada durante auditorías previas, resultados de procedimientos de auditoría, incluyendo fraude o error que puedan haberse encontrado, y fuente y confiabilidad de la información disponible. El resultado al final del proceso de auditoría se resume en el dictamen de auditoría, en este documento se presenta la opinión del auditor e indica de una manera razonable el estado de los estados financieros. (Sornoza Zamora \& Párraga Franco, 2016)

Como menciona Lagunes López N. (2012:211) un marco normativo ideal sobre la fiscalización de las cuentas públicas en México sería aquel en el cual sus conceptos sean definidos con claridad y cuyo lenguaje no sea demasiado técnico para hacerlo accesible a la ciudadanía; esto mismo debe exigirse a las entidades de fiscalización superior de los estados respecto al formato de presentación del Informe de Resultados de las cuentas públicas, al igual que una versión resumida del mismo y su publicación en los diarios de mayor difusión en cada uno de los estados y en la federación.

\section{Auditoria De Gabinete De La Cuenta Pública Municipal 2017 Del Estado De Sonora}

El objetivo de la auditoria de gabinete es la revisión a la información de la cuenta pública sobre la situación económica, las Finanzas Públicas, la Deuda Pública, los Activos y el Patrimonio Municipal correspondientes al ejercicio fiscal 2017, conforme al 
Programa Anual de Auditoría del Instituto Superior de Auditoria y Fiscalización, para lo cual se analiza y evalúa la información contenida en los informes en mención y se verifica además el cumplimiento legal de la presentación de la información contable, presupuestal, programática y Anexos, con el fin de conocer la calidad de la información financiera y que se haya realizado de conformidad a lo establecido en la Ley General de Contabilidad Gubernamental.

La Normatividad Aplicada está basada en los artículos 67 y 150 de la Constitución Política del Estado de Sonora y 1, 3, 6, 7, 17, 18, 23, 27, 28, 29, 30, 31, 34, 40, 41, 42 y 70 de la Ley de Fiscalización Superior para el Estado de Sonora, la presente auditoría se realizará utilizando las Normas de Auditoría Gubernamental consistentes en las Normas Profesionales de Auditoría del Sistema Nacional de Fiscalización y en su caso, las Normas Internacionales de Auditoría (NIAs) utilizadas por el Instituto Mexicano de Contadores Públicos.

A efectos de la ISSAI 1320, la importancia relativa o materialidad para la ejecución del trabajo referente a la cifra o cifras determinadas por el auditor, por debajo del nivel de la importancia relativa establecida para los estados financieros en su conjunto.

En el caso de las auditorías de la Información Financiera, la revisión privilegia el informe de Cuenta Pública proporcionado por el Municipio al ISAF por medio digital e impreso en las fechas establecidas en el artículo 7 de la Ley de Fiscalización Superior para el Estado de Sonora, toda vez que es la información más reciente y presenta cifras acumuladas.

No se determina el alcance en monto, toda vez que la evaluación es cualitativa de la información que presentan los formatos, sin embargo, se determina un alcance como sigue:
- Revisar que los saldos iníciales de todos los rubros de la Balanza de Comprobación del período comparativo deben corresponder a los de la Cuenta Pública del ejercicio inmediato anterior al ejercicio en revisión.

- Cotejar el importe del Presupuesto Estimado Original para Ingresos Propios contra el Autorizado en su Ley de Ingresos y Presupuesto de Ingresos para el Ejercicio Fiscal en revisión.

- Cotejar el importe del Presupuesto de Egresos Aprobado contra el Autorizado en su Presupuesto de Egresos para el Ejercicio Fiscal en revisión.

- Se revisará la Información Contable, Presupuestaria, Programática, Complementaria y Anexos, de acuerdo con lo señalado en los Procedimientos de Auditoría que se indican en el presente documento, verificando que cumplan con lo establecido en la Ley General de Contabilidad Gubernamental, los lineamientos emitidos por el Consejo Nacional de Armonización Contable (CONAC), las Recomendaciones generales de llenado de los formatos emitidas por el Consejo Nacional de Armonización Contable (CONAC) y en la Guía Informativa para la Presentación de la Información de la Cuenta Pública Municipal para el Ejercicio 2017.

Los procedimientos de revisión de los rubros de la Información Contable, Presupuestal, Programática, Complementaria y Anexos, que son objeto de esta revisión están en apego a las Normas Profesionales de Auditoría del Sistema Nacional de Fiscalización (NPASNF), las Normas Internacionales de Auditoría, la Planeación de la Auditoría, así como la importancia relativa o materialidad para la ejecución del trabajo. 


\section{Conclusión}

La auditoría y fiscalización gubernamental, es una forma de aplicar el control gubernamental en el ejercicio de los recursos públicos, que de acuerdo a la legislación vigente es responsabilidad de las Entidades de Fiscalización Superior; así como también los entes públicos deberán presentarle la información financiera, presupuestal, programática y complementaria de acuerdo a la normatividad establecida en las leyes aplicables a cada nivel de gobierno.

Por tanto, la tarea primordial de la Entidad Estatal de Fiscalización Superior de Sonora, consiste en revisar que los Municipios a través de sus Ayuntamientos cumplan con la obligación de entregar de forma clara, precisa y trasparente la información que estipulan la Ley General de Contabilidad Gubernamental, Ley de Disciplina Financiera de la Entidades Federativas y los Municipios, Ley de Fiscalización para el Estado de Sonora entre otros, debe contener la Cuenta Pública Municipal.

Para llevar a cabo esta evaluación existen diferentes tipo de Auditoría que el Órgano de Fiscalización ejecuta, una de ellas es la Auditoría Financiera de Gabinete, la cual es objeto de este estudio y cuyo enfoque es la revisión de la Situación Económica, las Finanzas Públicas, la Deuda Pública, los Activos y el Patrimonio Municipal informados en la Cuenta Pública Municipal, además del cumplimiento legal de la presentación de la información contable, presupuestal, programática y demás anexos, con la finalidad de conocer la calidad de la información financiera y que su elaboración haya sido de conformidad con lo establecido en la Ley General de Contabilidad Gubernamental.

\section{Referencias}

ASF. (2014). Normas Profesionales de Auditoría del Sistema Nacional de Fiscalización. México D.F.: INGRAFF S.A. de C.V.

Lagunes López, N. (2012). El marco legal de la fiscalización de las cuentas públicas del gobierno del estado de sonora, un estudio exploratorio. Revista legislativa de estudios sociales y de opinión pública , 209-237.

Lagunes López, O. N., \& Pineda Pablos, N. (2013). El arte de contar cuentos y rendir cuentas. La revisión de las cuentas públicas del Gobierno de Sonora, 2004-2010. Región y sociedad , 231-268.

López Hernández, A. M. (2013). Normas profesionales de la INTOSAI: directrices de auditoría. Aplicación a la actividad fiscalizadora de los Órganos Institucionales de Control Externo Autonómicos. Auditoría Pública , 9-24.

Pineda Pablos, N., García Figueroa, F., \& Rodríguez Camou, E. (2007). Democratización y rendición de cuentas, El caso de la revisión de las cuentas públicas municipales en Sonora. Gestión y Política Pública , 203-236.

Sornoza Zamora, J. C., \& Párraga Franco, S. (2016). Un acercamiento a los fundamentos teóricos y técnicos para desarrollar una auditoría integral. Revista Científica Dominio de las Ciencias , 276294.

Sour, L. (2017). Avances en la cantidad de la información financiera financiera del sector público en México a raíz de la LGCG. Contaduría y Administración , 419-441. 
Sour, L. (2011). El Sistema de Contabilidad Gubernamental que Mejorará la Transparencia y la Rendición de Cuentas en México. Transparencia y Privacidad, 33-57.

Sonora, C. d. (Última reforma 26-04-2018). Constitución Política del Estado de Sonora. Hermosillo, Sonora: Boletín del Estado de Sonora.

Sonora, I. S. (2018). Lineamientos que establecen el contenido y formularios para la elaboración de la cuenta pública municipal 2017. Hermosillo, Sonora: www.isaf.gob.mx

Sonora, H. C. (última Reforma B.O. 15-02-2018). Ley de Fiscalización Superior para el Estado de Sonora. Hermosillo, Sonora: Boletín Oficial del Estado de Sonora.

Unión, H. C. (Última Reforma 15-09-2017). Constitución Política de los Estados Unidos Mexicanos. Ciudad de México: Diario Oficial de la Federación.
Unión, H. C. (Ultima Reforma 30-01-2018). Ley de Disciplina Financiera de las Entidades Federativas y los Municipios. Ciudad de México: Diario Oficial de la Federación.

Unión, H. C. (Ultima Reforma DOF 30-01-2018). Ley General de Contabilidad Gubernamental. Ciudad de México: Diario Oficial de la Federación.

CONAC. (2018). Consejo Nacional de Armonización Contable. Recuperado de http://www.conac.gob.mx/ (Acuerdo por el que se armoniza la estructura de las cuentas públicas Última reforma publicada DOF 2902-2016)

ISAF. Recuperado de http://www.isaf.gob.mx Última consulta el 25 de junio del 2018 